\title{
Construction simplifiée d'un monde énergétique en 2050
}

Sandra Bouneau ${ }^{(1)}$, Sylvain David (1) (sdavid@ipno.in2p3.fr), Jean-Marie Loiseaux ${ }^{2}$

Olivier Méplan ${ }^{(2)}$ et Jacques Treiner ${ }^{(3)}$

(1) Institut de Physique Nucléaire, CNRS/IN2P3, Université Paris Sud, 91406 Orsay Cedex

(2) Laboratoire de Physique Subatomique et de Cosmologie, CNRS/IN2P3, INPG, Université Joseph Fourier, 38026 Grenoble Cedex 1

(3) Université Pierre et Marie Curie, 4 place Jussieu, 75005 Paris

L'énergie est un enjeu majeur du $21^{\mathrm{e}}$ siècle : le monde continue de consommer de plus en plus d'énergie, les ressources

en combustibles fossiles sont limitées, et nos émissions

de gaz à effet de serre risquent de mener à un dérèglement majeur du climat au cours du siècle à venir.

Dans ce cadre, de nombreuses études de prospective sont disponibles. Ces scénarios énergétiques sont, la plupart du temps, basés sur des modèles technico-économiques,

comportant de très nombreuses hypothèses, et des algorithmes d'optimisation complexes.

\section{Nous avons souhaité bâtir}

une représentation simplifiée et transparente de ce que pourrait être un monde énergétique en 2050, répondant à des hypothèses claires, afin de rendre les résultats compréhensibles et discutables par le plus grand nombre.
Le scénario qui découle de notre modèle cherche à satisfaire un compromis entre la stabilisation du réchauffement climatique et les besoins de développement des pays émergents et pauvres, en s'appuyant sur toutes les sources d'énergie dont nous disposons, fossiles, renouvelables et nucléaire. Par choix, le nombre de paramètres ajustables est donc limité. Cependant, il nous semble essentiel de tenir compte des hétérogénéités de consommation, que nous avons considérées non seulement entre les pays, mais au sein même de chaque nation. L'une des originalités de cette approche est donc de répartir la population totale de chaque nation en trois catégories de niveau de consommation : élevée, moyenne et faible. L'analyse des besoins et des sources disponibles en 2050 permet ensuite de bâtir un «mix » énergétique cohérent pour chacune des populations concernées. L'énergie nucléaire est ici utilisée comme variable d'ajustement pour compléter les besoins manquants après utilisation des autres sources (renouvelables, et fossiles avec émission ou séquestration de $\mathrm{CO}_{2}$ ), dont les potentiels ont été préalablement fixés à des valeurs délibérément optimistes.

\section{Décrire un scénario « 20 Gtep/an » en 2050}

La consommation mondiale d'énergie s'élevait en 2009 à près de $12 \mathrm{Gtep} / \mathrm{an}^{(\mathrm{a})}$, pour une consommation moyenne de 1,73 tep/habitant/an (voir tableau 1). Généralement, les inégalités de consommation sont décrites entre les grandes régions du monde, qui se répartissent en trois grands groupes de consommation : élevée (environ 3,5 à 7 tep/hab/an) pour les pays développés actuels (Europe, USA, ex-URSS, Pacifique), moyenne (1 à 1,5 tep/hab/an) pour les pays émergents (Asie et Amérique latine), et 0,5 à 0,75 tep/hab/an pour les pays les plus pauvres (Afrique subsaharienne).

Les études technico-économiques envisagent une consommation mondiale d'énergie en 2050 située entre 15 et 30 Gtep/an, alors que nous consommions au début des années 2000 environ 10 Gtep/an (13 Gtep/an en 2011). L'augmentation entre 2000 et 2011 suggérait une évolution vers les $30 \mathrm{Gtep} / \mathrm{an}$ en 2050, résultant notamment de la forte croissance des pays émergents comme la Chine, jusqu'à ce que la crise économique mondiale replace le monde sur une trajectoire

Tableau 1. Population et consommation d'énergie moyenne par habitant et totale, pour les huit grandes régions du monde, en 2009.

\begin{tabular}{|c|c|c|c|c|c|c|c|c|c|}
\hline 2009 & 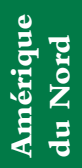 & $\frac{0}{2}$ & 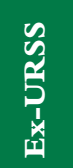 & $\begin{array}{l}0 \\
\frac{2}{2} \\
\frac{8}{2}\end{array}$ & $\begin{array}{l}\frac{1}{0} \\
\stackrel{0}{0} \\
\stackrel{0}{0} \\
\frac{0}{2}\end{array}$ & है & $\frac{0}{2}$ & $\frac{0}{2}$ & $\stackrel{\overrightarrow{7}}{\stackrel{+}{0}}$ \\
\hline $\begin{array}{l}\text { Population } \\
\text { (Mhab) }\end{array}$ & 341 & 162 & 279 & 534 & 210 & 583 & 3707 & 999 & 6816 \\
\hline $\begin{array}{c}\text { Consommation } \\
\text { d'énergie (tep/hab/an) }\end{array}$ & 7,1 & 4,0 & 3,4 & 3,3 & 2,4 & 1,3 & 1,1 & 0,75 & 1,73 \\
\hline $\begin{array}{c}\text { Énergie totale } \\
\text { consommée (Gtep/an) }\end{array}$ & 2,42 & 0,65 & 0,95 & 1,76 & 0,50 & 0,76 & 4,01 & 0,75 & 11,8 \\
\hline
\end{tabular}




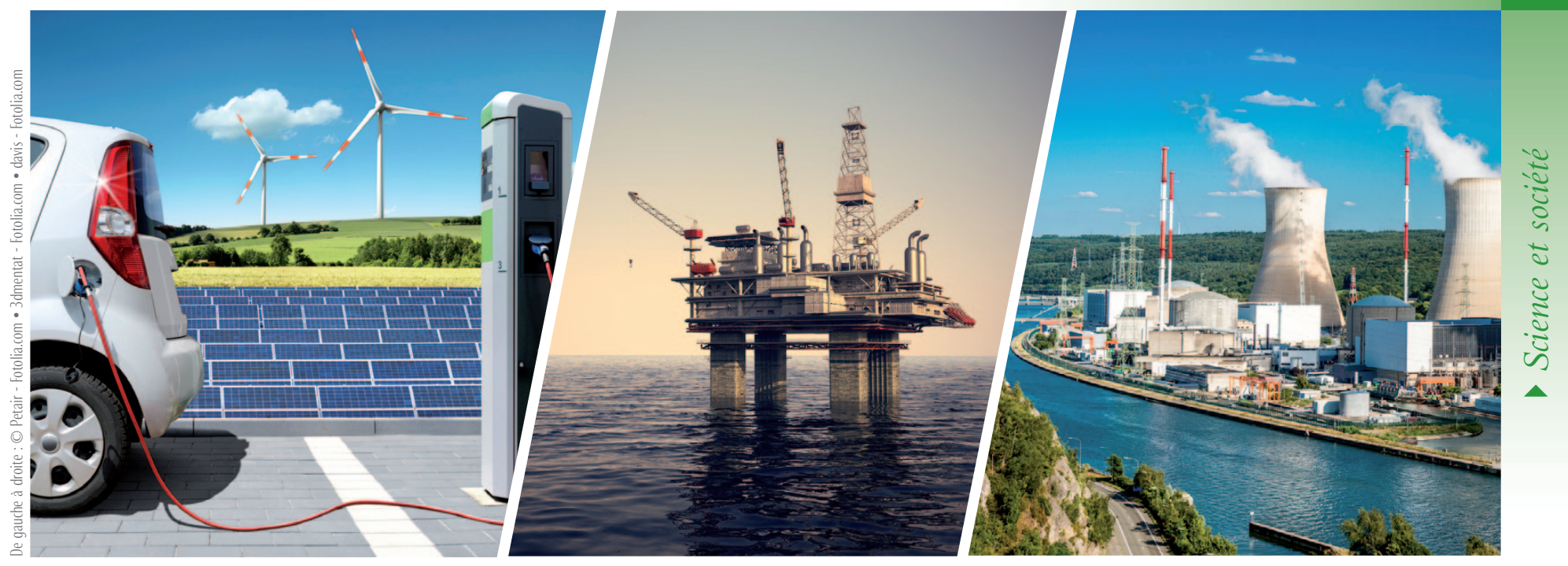

plus proche des « 25 Gtep/an en 2050 ». Nous avons choisi de fixer comme hypothèse de référence une consommation d'énergie mondiale en 2050 de 20 Gtep/an, qui apparaît comme une moyenne sobre des études de prospective. Il ne s'agit pas pour nous de décrire une trajectoire, mais le point d'arrivée en 2050, en commençant par répartir ces $20 \mathrm{Gtep} /$ an parmi les différentes populations prédites pour cette date.

\section{Répartition de la consommation}

Afin de prendre en compte les inégalités de consommation, que nous supposons persister dans le futur, nous considérons dans chaque région trois types de populations, $\mathrm{P}_{1}, \mathrm{P}_{2}$ et $\mathrm{P}_{3}$, de niveaux de consommation élevée, modérée et faible: $\mathrm{C}_{1}>\mathrm{C}_{2}>\mathrm{C}_{3}$. En effet, les entités économiques considérées ne sont pas homogènes : en Chine, par exemple, plusieurs centaines de millions d'habitants ont un niveau de vie comparable à celui des pays de l'OCDE, tandis que dans les campagnes reculées, les niveaux de consommation sont semblables à ceux de l'Afrique subsaharienne. Nous proposons donc une répartition de ces trois types de population dans les grandes régions du monde et, en fixant les clés d'inégalité ou rapports de consommation $\mathrm{C}_{1} / \mathrm{C}_{3}$ et $\mathrm{C}_{2} / \mathrm{C}_{3}$, nous obtenons immédiatement les consommations $\mathrm{C}_{1}, \mathrm{C}_{2}$ et $\mathrm{C}_{3}$. Enfin, connaissant pour chaque région du monde la répartition de $\mathrm{P}_{1}, \mathrm{P}_{2}$ et $\mathrm{P}_{3}$, il est ainsi possible de remonter à sa consommation d'énergie. Le schéma de la figure 1 résume la méthodologie employée.

Afin de répartir la population de chaque entité économique en 2050 dans ces trois groupes $\left(\mathrm{P}_{1}, \mathrm{P}_{2}\right.$ et $\left.\mathrm{P}_{3}\right)$, nous nous basons sur le niveau de développement actuel et sur les taux d'urbanisation prédits (projections de l'ONU), qui sont des indicateurs
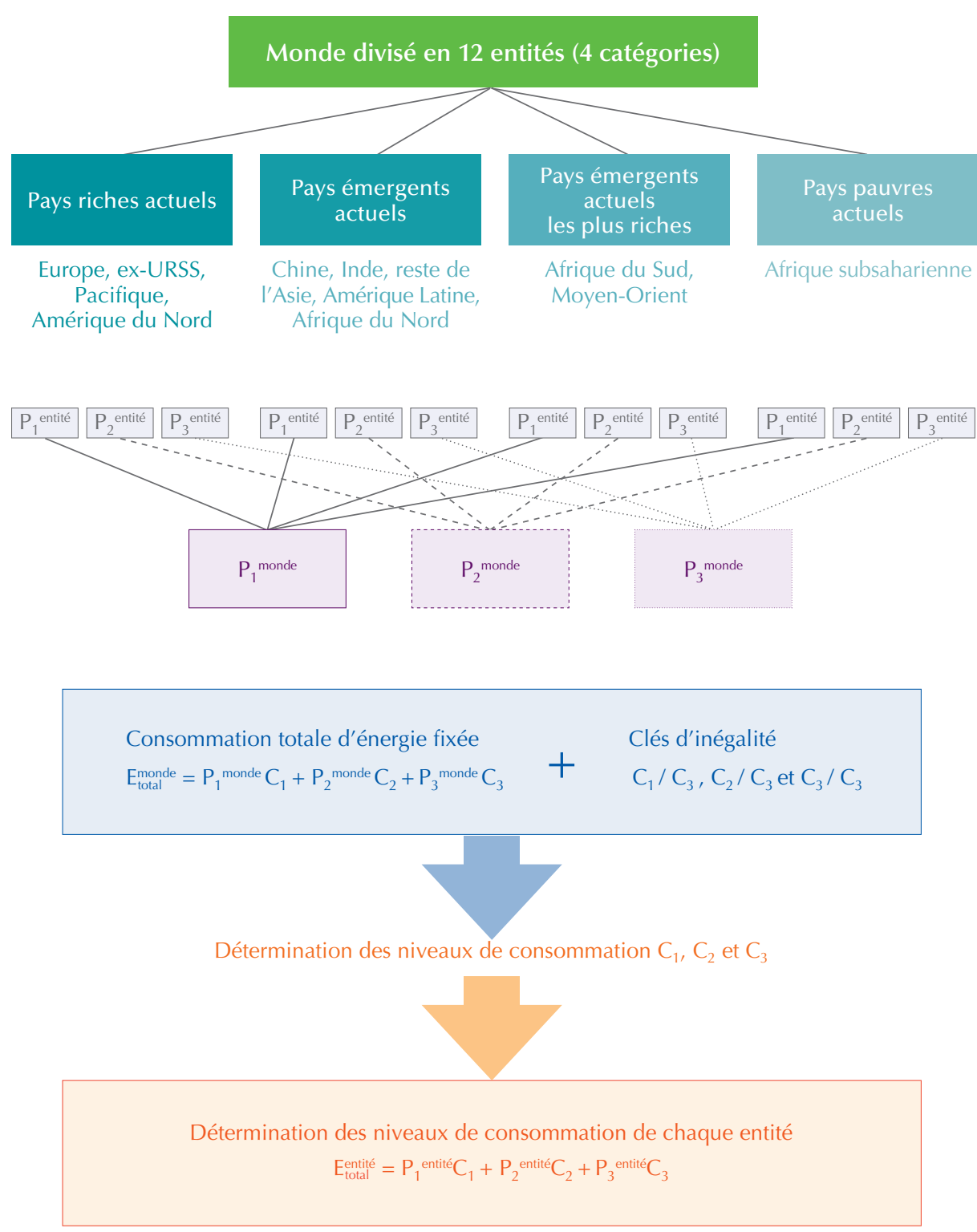

1. Schéma de principe de construction de la répartition de la consommation d'énergie totale mondiale (20 Gtep/an en 2050) entre les trois types de population $\mathrm{P}_{1}, \mathrm{P}_{2}$ et $\mathrm{P}_{3}$, et pour chaque grande entité économique. 
$>>$

robustes du niveau de développement d'un pays et de son niveau de consommation. Les villes sont le poumon économique des pays émergents et pauvres. Elles sont des centres d'échanges industriels, commerciaux et culturels, et les populations urbaines consomment beaucoup plus d'énergie que les populations rurales. Elles nécessitent des besoins concentrés en transport individuel ou collectif et en énergie. Nous proposons une répartition de référence, représentée dans le tableau 2.

Les résultats qui en découlent sont représentés dans le tableau 3.

Aujourd'hui, il y a plus d'un facteur 15 entre la consommation d'un habitant d'Amérique du Nord et celle d'un habitant des pays les plus pauvres d'Afrique subsaharienne. Même si des inégalités de consommation persistent, une lutte efficace contre le changement climatique nécessite un partage des efforts au niveau mondial, et donc une réduction drastique de ces inégalités. Cela nous conduit à fixer les clés d'inégalités de référence $\mathrm{C}_{1} / \mathrm{C}_{3}, \mathrm{C}_{2} / \mathrm{C}_{3}$ et $\mathrm{C}_{3} / \mathrm{C}_{3}$ à 4,2 et 1 . Ces rapports sont évidemment des paramètres modifiables du modèle.

\section{Projections de consommation}

À ce stade, la règle de somme : $\mathrm{P}_{1} \mathrm{C}_{1}+\mathrm{P}_{2} \mathrm{C}_{2}+\mathrm{P}_{3} \mathrm{C}_{3}=$

$\mathrm{C}_{3}\left[\mathrm{P}_{1}\left(\mathrm{C}_{1} / \mathrm{C}_{3}\right)+\mathrm{P}_{2}\left(\mathrm{C}_{2} / \mathrm{C}_{3}\right)+\mathrm{P}_{3}\left(\mathrm{C}_{3} / \mathrm{C}_{3}\right)\right]=$ $\mathrm{C}_{3}\left(4 \mathrm{P}_{1}+2 \mathrm{P}_{2}+\mathrm{P}_{3}\right)=20 \mathrm{GTep} / \mathrm{an}$, nous permet d'obtenir les valeurs de consommation (en tep/hab/an) suivantes : $\mathrm{C}_{1}=3,43, \mathrm{C}_{2}=1,72$ et $\mathrm{C}_{3}=0,86$.

Nous en déduisons alors la consommation moyenne par habitant de chacune des entités économiques (fig. 2).

Cette première étape permet de tirer quelques résultats saisissants :

- les pays riches doivent, d'ici 2050, réduire leur consommation moyenne d'énergie par habitant de $25 \%$ environ, ce qui à l'heure actuelle semble difficilement compatible avec une croissance économique de 1 à $2 \% / \mathrm{an}$;
- la Chine devrait rapidement stabiliser sa consommation d'énergie par habitant, ce qui semble également très difficile, étant donné les taux de croissance observés ces dernières années (de l'ordre de $10 \%$ /an pour le PIB comme pour la consommation d'énergie) ;

- les pays les plus pauvres atteindraient en 2050 une consommation de 1,3 tep/hab/an, ce qui peut être jugé très insuffisant, surtout lorsque l'on compare cette valeur aux niveaux de consommation actuelle des pays riches (3,5 à 7 tep/hab/an).

À ce stade, il semble que l'acceptabilité d'un scénario mondial "20 Gtep/an " peut être mise en question, tant il apparaît que les trajectoires actuelles de développement économique des pays riches, émergents et pauvres nous conduisent vers des valeurs plus élevées, au-delà de 25 Gtep/an. Nous conservons néanmoins cette première hypothèse, et étudions maintenant la façon dont les sources d'énergie du futur peuvent répondre à cette demande.

Tableau 2. Taux d'urbanisation prévu en 2050 et règle de répartition des populations urbaines $(U)$ et rurales $(R)$ de chaque entité économique entre les trois catégories de population $\mathrm{P}_{1}, \mathrm{P}_{2}$ et $\mathrm{P}_{3}$. Par exemple, en Chine, la moitié des urbains auront un niveau de consommation $\mathrm{C}_{1}$, l'autre moitié $\mathrm{C}_{2}$, et les ruraux $\mathrm{C}_{3}$.

\begin{tabular}{|c|c|c|c|c|c|c|c|c|c|}
\hline \multirow[t]{2}{*}{2050} & \multirow{2}{*}{$\begin{array}{l}\text { Pays } \\
\text { riches }\end{array}$} & \multicolumn{3}{|c|}{ Afrique } & \multirow{2}{*}{$\begin{array}{l}\text { Amérique } \\
\text { latine }\end{array}$} & \multicolumn{3}{|c|}{ Asie } & \multirow{2}{*}{$\begin{array}{c}\text { Moyen- } \\
\text { Orient }\end{array}$} \\
\hline & & Nord & Sud & Autres & & Chine & Inde & Autres & \\
\hline $\mathrm{P}_{1}$ & $\mathrm{U}+\mathrm{R}$ & $\mathrm{U} / 2$ & $\mathrm{U}$ & & $\mathrm{U} / 2$ & $\mathrm{U} / 2$ & $\mathrm{U} / 2$ & $\mathrm{U} / 2$ & $\mathrm{U}$ \\
\hline $\mathrm{P}_{2}$ & & $\mathrm{U} / 2$ & $\mathrm{R}$ & $\mathrm{U}$ & $\mathrm{U} / 2$ & $\mathrm{U} / 2$ & $\mathrm{U} / 2$ & $\mathrm{U} / 2$ & $\mathrm{R}$ \\
\hline $\mathrm{P}_{3}$ & & $\mathrm{R}$ & & $\mathrm{R}$ & $\mathrm{R}$ & $\mathrm{R}$ & $\mathrm{R}$ & $\mathrm{R}$ & \\
\hline $\begin{array}{l}\text { Taux d'urbanisation } \\
\text { en } 2050(\%)\end{array}$ & 80 & 71 & 80 & 59 & 89 & 73 & 54 & 63 & 80 \\
\hline
\end{tabular}

Tableau 3. Répartition prévue en 2050 des populations totales de chacune des entités économiques entre les trois catégories de population $\mathrm{P}_{1}, \mathrm{P}_{2}$ et $\mathrm{P}_{3}$.

\begin{tabular}{|c|c|c|c|c|c|c|c|c|c|c|}
\hline \multirow[t]{2}{*}{2050} & \multirow{2}{*}{$\begin{array}{l}\text { Pays } \\
\text { riches }\end{array}$} & \multicolumn{3}{|c|}{ Afrique } & \multirow{2}{*}{$\begin{array}{l}\text { Amérique } \\
\text { latine }\end{array}$} & \multicolumn{3}{|c|}{ Asie } & \multirow{2}{*}{$\begin{array}{l}\text { Moyen- } \\
\text { Orient }\end{array}$} & \multirow[t]{2}{*}{ Total } \\
\hline & & Nord & Sud & Autres & & Chine & Inde & Autres & & \\
\hline $\begin{array}{l}\text { Population totale } \\
\text { (Mhab) }\end{array}$ & 1428 & 322 & 57 & 1812 & 750 & 1305 & 1692 & 1558 & 378 & 9303 \\
\hline $\mathrm{P}_{1}$ & 1428 & 114 & 45 & & 333 & 479 & 459 & 492 & 302 & 3653 \\
\hline $\mathrm{P}_{2}$ & & 114 & 12 & 1071 & 333 & 479 & 459 & 492 & 76 & 3036 \\
\hline $\mathrm{P}_{3}$ & & 94 & & 741 & 84 & 347 & 774 & 574 & & 2613 \\
\hline
\end{tabular}




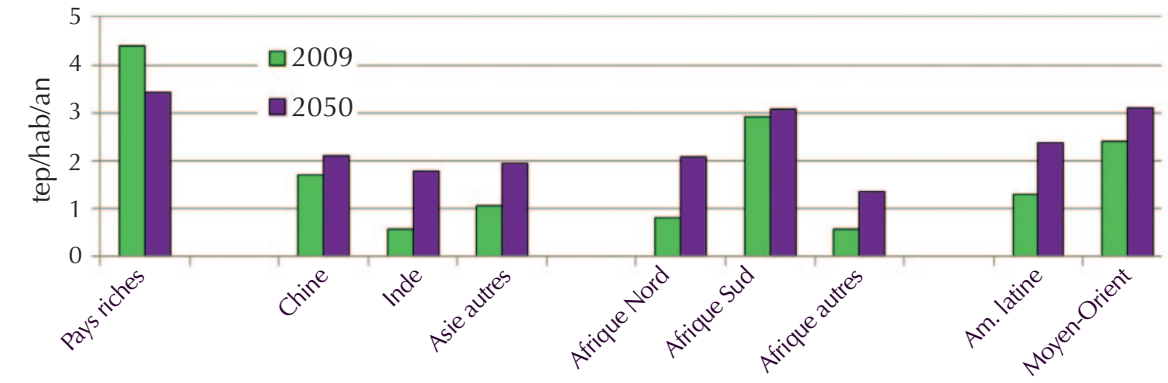

2. Consommation d'énergie par habitant en 2009 et (calculée dans le modèle) en 2050, dans les grandes régions du monde.

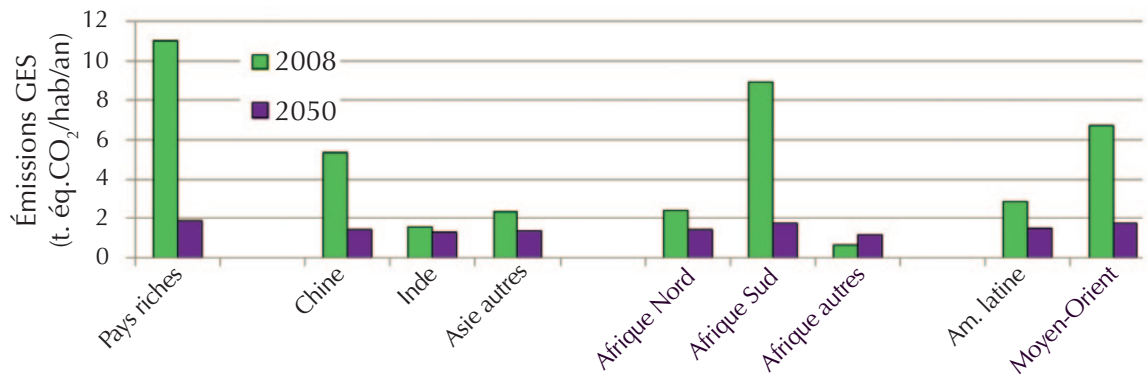

3. Émission moyenne de gaz à effet de serre (GES, en équivalent $\mathrm{CO}_{2}$ ) par habitant, pour les grandes régions du monde, en 2008 et (évaluée par le modèle) en 2050.

\section{Construction d'un bouquet énergétique en 2050}

Le bouquet énergétique se bâtit pour chaque type de population, en s'assurant de l'adéquation des sources et des besoins, répartis en quatre catégories : transport, chaleur industrielle (haute température), chaleur pour le résidentiel/tertiaire (basse température) et électricité.

\section{La contrainte climatique}

La première étape consiste à évaluer les consommations de combustibles fossiles avec émission de $\mathrm{CO}_{2}$, notées $\mathrm{F}_{1}, \mathrm{~F}_{2}$ et $\mathrm{F}_{3}$ (en tep/hab/an), pour chacune des populations $\mathrm{P}_{1}, \mathrm{P}_{2}$ et $\mathrm{P}_{3}$. Les émissions annuelles s'élèvent aujourd'hui à 29 Gt.éq. $\mathrm{CO}_{2}{ }^{(b)}$ pour les usages énergétiques (soit 4,5 t.éq. $\mathrm{CO}_{2}$ par habitant, en moyenne), et nous fixons

\section{Les besoins en énergie par type de population}

Afin de construire un «mix » énergétique cohérent et le plus réaliste possible, il s'agit d'assurer l'adéquation des sources d'énergie envisagées et des besoins, pour chaque type de population. Si l'on suppose que pour les pays riches, émergents et pauvres, la répartition de l'énergie totale entre les quatre secteurs de consommation sera la même en 2050 qu'actuellement, on obtient les consommations indiquées dans le tableau 4.

Le principe de la construction consiste à "remplir " au fur et à mesure les quatre types de besoins, pour chaque type de population, en ayant recours aux diverses sources d'énergie disponibles. Chaque source s'applique à un ou des besoins spécifiques et ne peut en couvrir la totalité. Les besoins en énergie qu'il reste à couvrir définissent une "énergie manquante ", qu'il faut combler avec les autres sources d'énergie. La méthodologie est décrite ci-dessous.

- Les combustibles fossiles avec émission de $\mathrm{CO}_{2}$ sont principalement réservés au transport, car il s'agit du poste où il sera difficile de trouver des alternatives et de stocker le $\mathrm{CO}_{2}$ émis (le reste est réparti au prorata des autres besoins). Cette étape définit une "énergie manquante 1 " (EM1), qu'il reste à fournir par d'autres sources pour chaque population et chaque type de besoin.

- On réserve la majeure partie de la biomasse traditionnelle pour les populations rurales, de type $\mathrm{P}_{3}$. La biomasse est utilisée pour la production de chaleur à haute et basse température, et également pour l'électricité des populations rurales $\left(\mathrm{P}_{3}\right)$. Les biocarburants sont utilisés pour boucler les besoins en transport des populations rurales, puis répartis au prorata des besoins manquants entre $\mathrm{P}_{1}$ et $\mathrm{P}_{2}$ (EM2).

Tableau 4. Répartition des 20 Gtep/an (consommation mondiale estimée en 2050) entre les quatre grands secteurs de consommation, selon les trois groupes de population $\mathrm{P}_{1}, \mathrm{P}_{2}$ et $\mathrm{P}_{3}$.

\begin{tabular}{|c|c|c|c|c|}
\hline & $\begin{array}{c}\text { Transport } \\
\text { \% (Gtep/an) }\end{array}$ & $\begin{array}{c}\text { Industrie } \\
\text { \% (Gtep/an) }\end{array}$ & $\begin{array}{c}\text { Résidentiel/tertiaire } \\
\text { \% (Gtep/an) }\end{array}$ & $\begin{array}{c}\text { Electricité } \\
\text { \% (Gtep/an) }\end{array}$ \\
\hline $\mathrm{P}_{1}$ & $25(3,14)$ & $20(2,51)$ & $15(1,88)$ & $40(5,02)$ \\
\hline $\mathrm{P}_{2}$ & $25(1,30)$ & $25(1,30)$ & $20(1,04)$ & $30(1,56)$ \\
\hline $\mathrm{P}_{3}$ & $20(0,45)$ & $15(0,34)$ & $40(0,90)$ & $25(0,56)$ \\
\hline
\end{tabular}


$\gg>$

- On distribue les énergies renouvelables non productrices d'électricité au prorata des besoins manquants des populations $\mathrm{P}_{1}, \mathrm{P}_{2}$ et $\mathrm{P}_{3}$, pour la production de chaleur à haute et basse température (EM3).

- On considère ensuite la cogénération de chaleur et d'électricité, en utilisant des combustibles fossiles avec séquestration $\mathrm{du} \mathrm{CO}_{2}$. Compte tenu des besoins manquants, nous choisissons de combler une partie de la chaleur industrielle par du nucléaire dédié exclusivement à la production de chaleur haute température.

- À ce stade, tous les besoins manquants en transport et chaleur (EM4) doivent être reportés sur l'électricité. Ce transfert se fait de manière à minimiser la consommation électrique, en utilisant notamment des pompes à chaleur pour une grande partie du chauffage du résidentiel/tertiaire.

- La demande totale en électricité est alors assurée par les énergies renouvelables électriques (réparties au prorata des besoins manquants entre $\mathrm{P}_{1}, \mathrm{P}_{2}$ et $\mathrm{P}_{3}$ ), puis par la part restante des fossiles avec séquestration de $\mathrm{CO}_{2}$ (sans cogénération) en s'assurant qu'à ce stade, tous les besoins électriques pour la population $\mathrm{P}_{3}$ soient assurés. Enfin, le nucléaire est utilisé pour combler les besoins (concentrés) des populations urbaines $\mathrm{P}_{1}$ et $\mathrm{P}_{2}$.

\section{Le potentiel des sources alternatives}

Pour les énergies renouvelables, nous nous basons sur des estimations parmi les plus optimistes rencontrées dans la littérature ${ }^{(\mathrm{d})}$, résumées dans le tableau 5.
En ce qui concerne la séquestration de $\mathrm{CO}_{2}$, le potentiel estimé le plus optimiste est de l'ordre de $12 \mathrm{Gt}$ de $\mathrm{CO}_{2}$ séquestrées chaque année, ce qui correspond à une consommation d'énergie de l'ordre de 3,7 Gtep/an, dont 2,7 Gtep/an sont considérés en cogénération $^{(\mathrm{e})}$, et $1 \mathrm{Gtep} /$ an sans cogénération.

\section{Le bouquet énergétique}

La figure 4 résume la première partie de la construction du "mix " énergétique, avant transfert des besoins manquants (EM5) sur l'électricité. Il apparaît clairement que, compte tenu des potentiels estimés des différentes sources pour les transports et pour la chaleur, une grande partie de ces besoins (20 à $30 \%$ ) pour les populations $\mathrm{P}_{1}$ et $\mathrm{P}_{2}$ doivent être transférés sur l'électricité. Pour $\mathrm{P}_{3}$, un tel transfert n'est pas nécessaire, du fait de la faible consommation totale et de l'utilisation massive de la biomasse.

La figure 5 résume la production d'électricité, après transfert de l'énergie manquante EM5, pour chaque type de population. Par hypothèse, les combustibles fossiles avec émission de $\mathrm{CO}_{2}$ sont très peu utilisés (réservés au transport), la biomasse est uniquement envisagée pour les populations rurales $\left(\mathrm{P}_{3}\right)$ et le nucléaire uniquement pour les populations urbaines $\left(\mathrm{P}_{1}\right.$ et $\left.\mathrm{P}_{2}\right)$.

Enfin, les figures 6 et 7 donnent le bouquet énergétique global et le bouquet électrique détaillé en 2050 pour chacune des régions du monde considérées dans cette construction.

$\mathrm{Au}$ vu de ces résultats, quelques grandes lignes peuvent être mises en exergue.
Tableau 5. Potentiel estimé (en Gtep/an) des énergies renouvelables pour 2050, et types d'usage (indiqués par des croix). Notons que, par rapport aux productions de 2008, l'éolien est multiplié par 20 et le photovoltaïque par environ 2000.

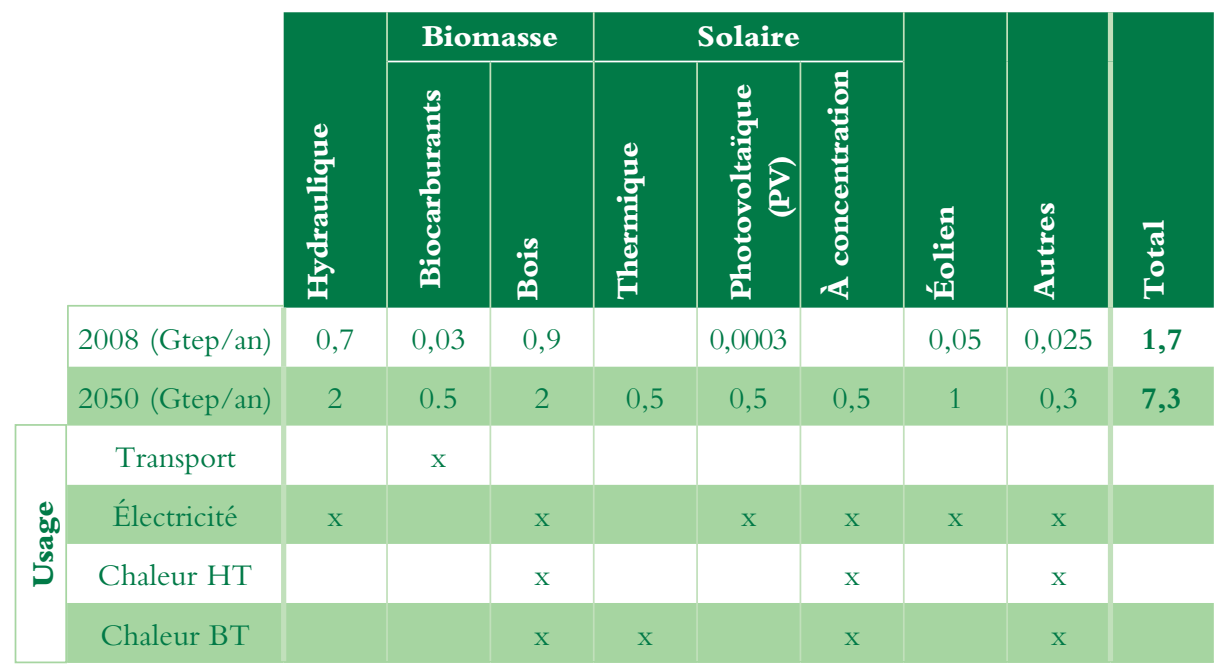

- La consommation des ressources traditionnelles (par habitant), énergies fossiles avec émission de $\mathrm{CO}_{2}$ et biomasse, est assez homogène au niveau mondial. La structure de consommation differe sur les nouvelles énergies, renouvelables électriques et nucléaire, d'autant plus utilisées qu'un pays est développé.

- Chaque région a recours à la séquestration du $\mathrm{CO}_{2}$ (de 0,25 à 0,66 tep/hab/an).

- Le nucléaire se déploie essentiellement dans les pays émergents et riches, à hauteur de 0,4 à 1,1 tep/hab/an $(0,28$ pour l'Afrique subsaharienne), et atteint au total 5,42 Gtep/an (25\% de l'énergie mondiale), soit 8 fois plus qu'aujourd'hui. Cela correspond néanmoins à une nucléarisation bien moindre que le cas actuel de la France $(\sim 6500 \mathrm{kWh} / \mathrm{hab} / \mathrm{an}$ en France en 2010, contre 4300 pour les pays riches, et de l'ordre de 1600 à 2000 en Asie pour la production électrique en 2050).

- Dans chaque région du monde, la part de l'électricité intermittente atteint environ $15 \%$ de l'électricité totale. Cette part atteint $50 \%$ de celle de l'électricité modulable (barrages hydrauliques, fossiles sans cogénération), ce qui est bien supérieur à ce que peut absorber actuellement un réseau électrique (de l'ordre de $20 \%$ ). Ceci donne un ordre de grandeur du défi majeur de la gestion de l'intermittence dans le futur. Le stockage de l'électricité, ainsi qu'une meilleure modulation de la production électrique par le nucléaire seraient des façons de gérer une partie de ce problème.

\section{Conclusion}

Nous avons proposé une construction simplifiée d'un monde énergétique en 2050, qui répond à des contraintes quantifiées, comme une consommation d'énergie mondiale de 20 Gtep/an et une réduction par deux des émissions de gaz à effet de serre. Elle permet de discuter des grandes lignes du débat sur l'avenir énergétique de la planète. Compte tenu de la réduction des inégalités de consommation envisagée, un scénario " 20 Gtep/an » apparâtt comme très sobre, et peut-être inacceptable, tant par les pays riches que par les populations les plus défavorisées. Ce travail met également en évidence, de manière quantifiée, qu'il semble impossible d'assurer une croissance économique mondiale en répondant à la contrainte climatique et en 


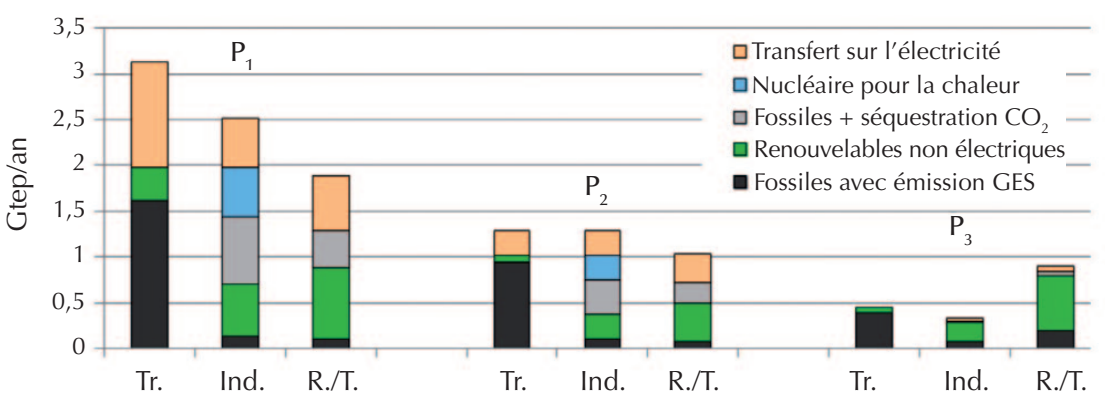

4. Construction du bouquet énergétique pour les besoins en transport (Tr.), chaleur pour l'industrie (Ind.) et le résidentiel/tertiaire (R./T.), pour chaque type de population. La partie supérieure des barres (en orange) représente l'énergie manquante qu'il faut transférer sur l'électricité

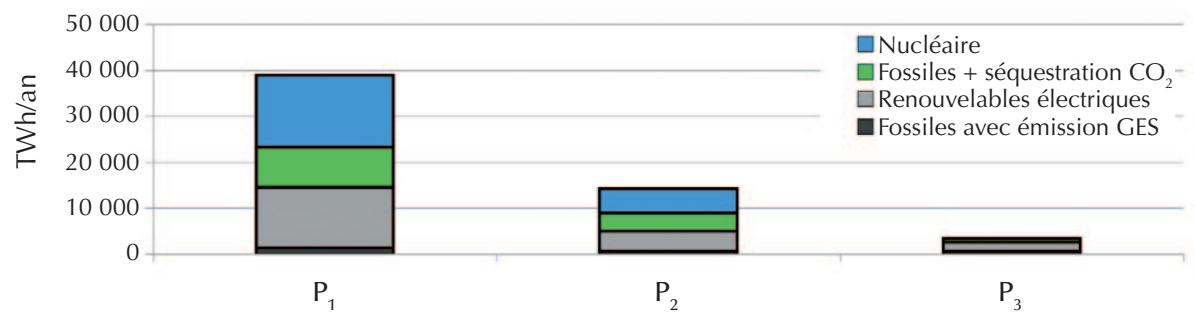

5. Construction du bouquet électrique correspondant aux besoins totaux en électricité (après transfert) pour chaque type de population, $\mathbf{P}_{1}, \mathbf{P}_{2}$ et $\mathbf{P}_{\mathbf{3}}$. Nous avons choisi la convention $10000 \mathrm{TWh}=2,2 \mathrm{Gtep}$.

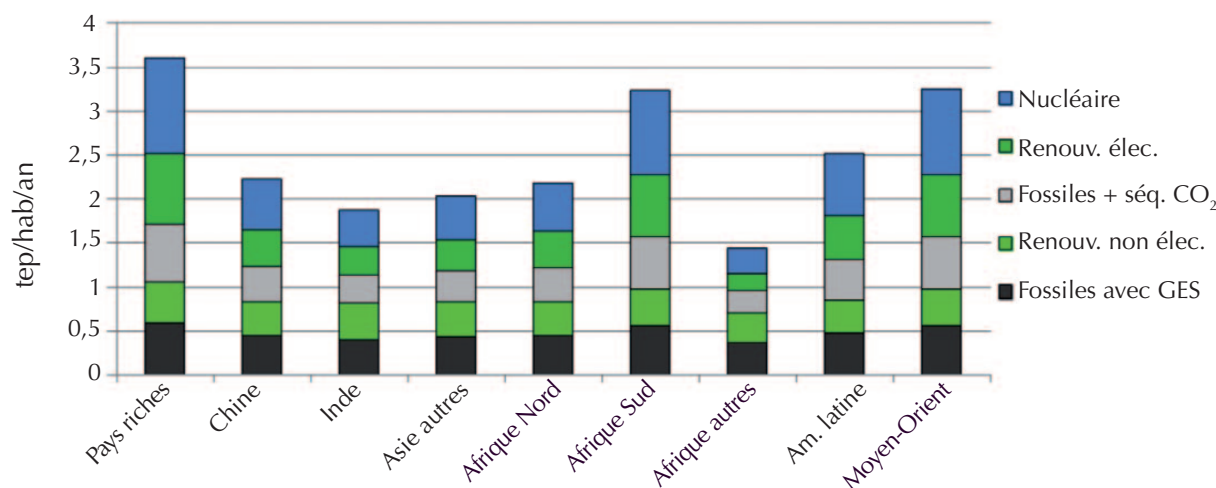

6. Bouquet énergétique obtenu en 2050 pour chacune des grandes régions du monde.

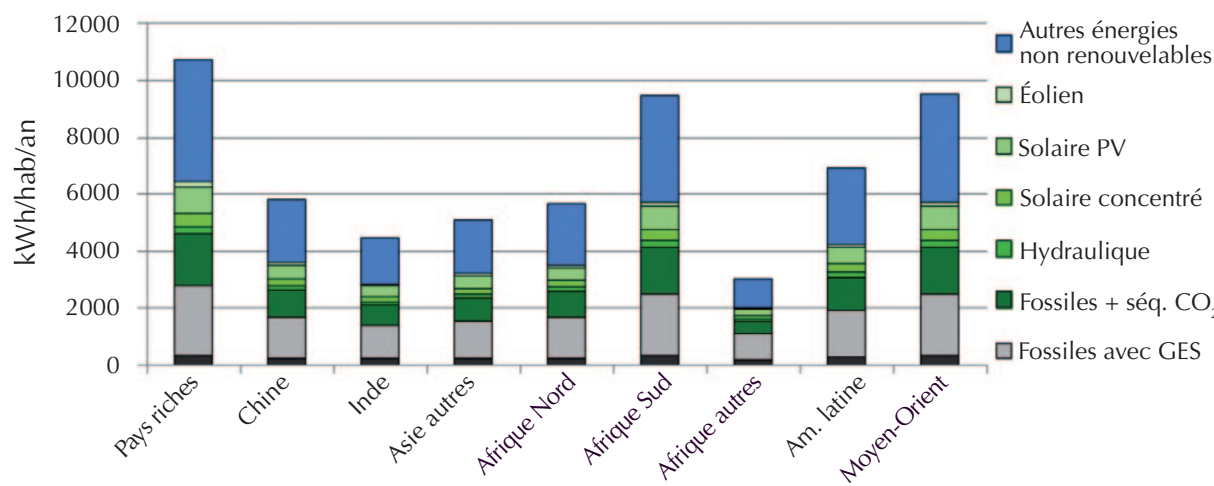

7. Bouquet électrique obtenu en 2050 pour chacune des grandes régions du monde. sortant du nucléaire. Les résultats obtenus ne décrivent pas une trajectoire énergétique ; ils doivent être vus comme des outils pour caractériser un monde énergétique et aider à définir les grandes priorités en matière de politiques publiques et de recherche et développement. Au vu de ces résultats, l'alternative entre sobriété couplée aux énergies renouvelables d'une part et le nucléaire d'autre part, ne semble plus au niveau de l'enjeu du développement et du climat. De même, la séquestration du $\mathrm{CO}_{2}$ paraît incontournable pour assurer une réduction d'un facteur deux des émissions mondiales, même avec un recours massif au nucléaire et aux renouvelables. Un autre exemple, le manque de sources suffisantes pour les transports et la production de chaleur, demande à reporter une part significative de ces besoins sur l'électricité, à l'heure où les normes françaises pour le bâtiment favorisent le chauffage au fuel ou au gaz et se veulent impossibles à tenir pour le chauffage électrique.

Enfin, la simplicité et la transparence de cette construction doivent permettre à tous de pouvoir discuter les hypothèses ou la méthodologie, et de mener des études de sensibilité aux hypothèses, afin de tester la robustesse des différents résultats obtenus. Elles permettent également d'initier une discussion interdisciplinaire sur ce sujet complexe, faisant intervenir quasiment toutes les disciplines de recherche, tant en sciences sociales qu'en sciences de la matière.

(a) La "tonne équivalent pétrole " (tep) est une unité de mesure de l'énergie, utilisée pour comparer les énergies entre elles. Elle correspond à l'énergie produite par la combustion d'une tonne de pétrole "moyen ", ce qui représente environ 42 GJ ou $11670 \mathrm{kWh}$.

(b) La "tonne équivalent $\mathrm{CO}_{2}$ " (t.éq. $\mathrm{CO}_{2}$ ) est une unité qui permet de donner une équivalence, en terme de production d'effet de serre, entre les différents gaz à effet de serre et le dioxyde de carbone. Cela permet d'évaluer globalement l'effet de serre engendré par différentes sortes d'émissions.

(c) GIEC 2007, Bilan 2007 des changements climatiques. Contribution des Groupes de travail I, II et III au 4 e rapport d'évaluation du Groupe d'experts intergouvernemental sur l'évolution du climat [Equipe de rédaction, R.K. Pachauri et A. Reisenger]. GIEC, Genève, Suisse.

(d) Les estimations sont essentiellement basées sur le rapport du World Energy Outlook - WETO H2, European Commission, EUR22038,

http://ec.europa.eu/research/energy/gp/gp_pu/ article_1100_en.htm

(e) La cogénération consiste à produire de l'électricité dans une machine thermique et à récupérer la chaleur rejetée pour le chauffage des habitations ou des besoins industriels. On optimise ainsi la consommation de combustible fossile. 